\title{
Novel Imaging and Clinical Manifestations of Ndmsba Disorder Caused by a Homozygous Missense Variant of Plaa
}

\section{Ali Dehghani}

Tarbiat Modares University Faculty of Medical Sciences

\section{Ehsan Razmara}

Tarbiat Modares University Faculty of Medical Sciences

Maryam Rasoulinezhad

Tehran University of Medical Sciences

Fatemeh Bitarafan

deparment of medical genetic, Dena labratory

Ali Reza Tavasoli

Tehran University of Medical Sciences

Masoud Garshasbi ( $D$ masoud.garshasbi@modares.ac.ir)

Tarbiat Modares University Faculty of Medical Sciences https://orcid.org/0000-0002-5508-7903

\section{Research}

Keywords: NDMSBA, phospholipase A-2-activating protein, PLAA, PUL domain, WES

Posted Date: April 13th, 2021

DOI: https://doi.org/10.21203/rs.3.rs-407675/v1

License: (c) (i) This work is licensed under a Creative Commons Attribution 4.0 International License. Read Full License 


\section{Abstract}

\section{Background}

Phospholipase A-2-activating protein (PLAP) has essential roles in biological pathways. Neurodevelopmental disorder with progressive microcephaly, spasticity, and brain anomalies (NDMSBA) is a complex neurodevelopmental disease caused by defects in the PLAA gene (MIM: 603873). Herein, we aimed to detect the potential genetic factors contributing to the NDMSBA phenotype in a 2.5-year-old affected male in an Iranian consanguineous family.

Results

After meticulously performing neuroimaging and clinical examinations, due to heterogeneity of neurodevelopmental diseases, the proband was subjected to paired-end whole-exome sequencing (WES). The brain magnetic resonance imaging (MRI) revealed lissencephaly, polymicrogyria, severe subcortical, deep and deep white matter signal abnormalities, thinning of the corpus callosum, and severe vermis atrophy. Interestingly, we detected a novel homozygous missense variant, NM_001031689.3:c.2264A>G;p.(Asp755Gly) in the PLAA gene. To the best of our knowledge, this variant is the second one identified within the PUL domain (PLAP, Ufd3p, and Lub1p) of PLAP and also the sixth reported variant throughout the PLAA gene. In silico analyses underscored the pathogenicity of the variant.

Conclusions

The present study demonstrated severe cerebral and cerebellar white matter signal abnormalities, hypertelorism, strabismus, and drooling in the proband as the novel manifestation of NDMSBA that in turn caused by a novel likely pathogenic missense variant. Further studies are required to confirm how this variant contributes to NDMSBA.

\section{Background}

Neurodevelopmental disorder with progressive microcephaly, spasticity, and brain anomalies (NDMSBA) is an autosomal recessive disorder. Affected individuals are characterized by spastic quadriparesis, the infantile-onset of progressive microcephaly [1], failure to thrive, cognitive, and motor impairments [2]. Other clinical manifestations may include seizure that has been reported in $73.3 \%$ of affected patients and optic atrophy [3]. Furthermore, brain imaging may reveal impaired myelination and white matter signal lesions, and structural anomalies such as gyral abnormalities (lissencephaly and polymicrogyria), thin corpus callosum, cerebral or cerebellar atrophy [2]. Like almost any other neurodevelopmental disorder, NDMSBA is diagnosed by evaluating the presence of manifestations or abnormal behaviors in patients and may also be confirmed by genetic analysis.

NDMSBA is a rare genetic condition associated with homozygous loss of function mutations in the Phospholipase A2activating (PLAA; OMIM: 603873) that encodes ubiquitin-binding protein [1]; up to now, only six mutations have been recorded in PLAA that are associated with this disorder. The PLAA gene, also known as DOA1, contains 14 exons and extends about $44 \mathrm{kbp}$ on chromosome 9p21.2. According to the Human Protein Atlas [4], extensive-expression of PLAA is detectable within the cytoplasm and nucleus, underscoring the potential vital roles of the encoded proteinPhospholipase A-2-activating protein (PLAP) -in cellular events. PLAP plays a pivotal role in regulating the inflammatory response through activating the phospholipase $A 2$, which catalyzes the release of arachidonic acid from membrane phospholipids [5]. PLAP is also a contributing factor to ubiquitin-dependent protein degradation via the ubiquitinproteasome system and lysosomal degradation, and the turnover of synaptic membrane proteins important in neurotransmission and synaptic function [6]. Indeed, the PLAA family ubiquitin-binding domain (PFU) in the central part of PLAP binds to ubiquitin and a C-terminal PUL (PLAP, Ufd3p, and Lub1p) domain, which consists of six armadillo 
domains that interact with the p97 enzyme which is involved in protein turnover and degradation [7, 8]. WD40 betapropeller and PFU domain bind to ubiquitin with high-affinity and low-affinity, respectively $[9,10]$.

Ubiquitin signaling has been identified to play a critical role in synapse development and plasticity $[3,11]$. Interruption of the Ub-mediated signaling pathway causes a variety of diseases such as cancer [12], immune deficiency [13], diabetes [14], and neurodegeneration [15]; the proteotoxic aggregation of ubiquitinylated proteins can result in neurodegenerative disorder [16]. It has been properly documented that PLAP is essential for neural function using two different mechanisms: firstly, the adjustment of post-endocytic trafficking of signaling receptors, which is imperative for neural development, and secondly sorting synaptic vesicle components during recycling that is essential for synaptic function [3]. Ubiquitin-dependent endolysosomal proteostasis at the synapse is also an indispensable factor for neuronal activity [3].

Herein, we analyzed the clinical features of a male patient from a consanguineous Iranian family, affected by NDMSBA disease. Using Whole-exome sequencing (WES), we introduced a novel variant, NM_001031689.3:c.2264A > G;p. (Asp755Gly), in the PLAA gene associated with NDMSBA. To the best of our knowledge, this variant is the second identified variant within the PUL domain (PLAP, Ufd3p, and Lub1p) of PLAP and also the sixth reported one throughout the PLAA gene. Moreover, novel neuroimaging and clinical features are reported in association with NDMSBA.

\section{Results}

\section{Clinical presentation}

A 2.5-year-old male born to a consanguineous family was referred to Myelin Disorder Clinic, Tehran, Iran due to neurodevelopmental delay in association with strabismus and esotropia; these conditions were since birth-time. According to initial neurological, imaging, and metabolic evaluations, a genetically-based intellectual disability was suspected. The proband (IV.2) was the second child of a family in which the family history of seizure was obvious, i.e. his aunt (III.1) suffered from seizure without remarkable details (Fig. 1a). However, the family never had any history of abnormal movements and seizures. His seven-year-old brother was apparently healthy.

The proband was born in a full-term pregnancy without any significant complications during prenatal and perinatal periods. His birth-time weight was reported $3.85 \pm 0.01 \mathrm{~kg}$ that was in the normal range [17], while his head circumference $(\mathrm{HC})$ was measured at $36.5 \pm 0.1 \mathrm{~cm}$ (in comparison to $35.13 \pm 1.45 \mathrm{~cm}$ as the average in the Iranian population [18]). Regarding the developmental milestones, he had a global developmental delay at 10 months of age as severe as he did not have complete neck holding, unable to sit without any aids, had limited fix and follow, got poor cognition inappropriate for his chronological age using Denver Developmental Screening Test (DDST), and had drooling. Besides that, on the first physical examination at this age, his weight was determined $7.0 \mathrm{~kg}$ (percentile 0.7 ; z-score: -2.4) and HC was $43 \mathrm{~cm}$ (3rd percentile; z-score: -1.9); and he was manifesting somehow dysmorphic features including protruding ears, micrognathia, high arch palate, and hypertelorism.

On the last physical examination era-that was performed at the age of 2 years-head circumference and weight were measured $47.0 \pm 0.1 \mathrm{~cm}$ (14th percentile) and11.0 kg (percentile 23.33; z-score: -0.7 ), respectively. It seemed that the gross motor function milestones had been improving; although the patient was able to sit for a few minutes with assistance, he had no parachute reflex. In sum, the disease course was developed slowly without any neurologic regression. The patient's gross motor ability was calculated 3/5 based on the Gross Motor Function Classification System (GMFCS) score. Ophthalmic examination revealed persistent strabismus with remarkable esotropia, limited fix and follow and end gaze nystagmus, and normal pupils' reaction to light. His gag reflex was not satisfactory as he had drooling. Regarding the level of the patient's cooperation, further cranial nerves examination was normal. No abnormal movement or spasticity was recognized. Deep tendon reflexes were normal and plantar reflexes were bilaterally

Page 3/16 
downward. The skin was normal without any congenital or acquired rash. In general, the proband had poorly achieved developmental milestones at the age of 2 years. By the time the proband was subjected to be prescribed carnitine solution, omega 3 syrup, and supplementary vitamin E and D in addition to rehabilitation program during the follow-up period resulted in some progression in motor milestone.

All basic metabolic screening tests including thyroid function tests, urine organic acids profile, metabolic screen (MS/MS), serum ammonia, and lactate as well as additional ophthalmologic examinations including retinal, anterior, and posterior segments were normal. The first brain magnetic resonance imaging (MRI) that was done at the age of 18 months showed lissencephaly, polymicrogyria, extensive subcortical, periventricular and deep white matter signal abnormalities, thinning of the corpus callosum, and severe vermis atrophy (Fig. 1b-g).

\section{Genetic analysis}

Paired-end WES was performed for the DNA sample of the proband. After alignment and SNV calling, in total, 81,716 variants were found in the proband. To decrease the number of the variants as much and accurate as possible, the alleles with minor allele frequency $>1 \%$ were excluded using dbSNP150 [19, 20], 1000 Genomes Project [21], Exome Sequencing Project [22], and the genome aggregation database (gnomAD) [23]. According to variant functionality scores provided by Sorting Intolerant from Tolerant (SIFT) [24], PolyPhen-2 [25], and MutationTaster [26], the remained variants were prioritized. Furthermore, to this end, we used the important clinical features observed in the proband (e.g. lissencephaly and other brain anomalies) using ClinVar (https://www.ncbi.nlm.nih.gov/clinvar) [27], Phenolyzer [28], and Face2Gene [29], only one relevant variant, NM_001031689.3:c.2264A > G;p.(Asp755Gly), was identified in the PLAA gene (Fig. 2a). We also checked the allele frequency in the Iranian population employing Iranome (http://www.iranome.ir/) [30] as a local database. The variant had not been reported in the Iranian population or any other sub-populations.

We analyzed the DNA samples from other available family members by Sanger sequencing that in turn confirmed the cosegregation of the missense variant of the PLAA gene. By assuming that the contributing genetic factor can be traced in the autosomal dominant mode of inheritance, we reanalyzed WES data, but no variant resulted from this supposition though (Fig. 2b).

\section{In silico predictions}

To evaluate the possible pathogenicity of the detected variant, locating on exon 14 of the PLAA gene, different databases were used including SIFT, PolyPhen-2, MutationTaster, and Provean (Fig. 3a) [31]. The conservation analyses performed at the nucleotide level by using the '2-Way Pseudogene Annotation Set' from the UCSC genome browser database [32] indicated that the variant was located in a highly conserved area in a wide range of species, specifically advanced cognitive animals and primates. Also, Consurf revealed that the variant was in a variable region in protein level (Consurf score 1.0) (Fig. 3b). PDB file was built using protein structure homology-modeling online servers Phyre2 [33] and SWISS-MODEL [34]. The difference in flexibility and stability of wild-type and mutant residues suggest an unstable PLAP, which was verified and obtained using l-Mutant 3.0 sever [35] $(\Delta G<0)$. Finally, the possible effects of novel variant on PLAP and the protein structures were depicted by PyMOL [36] (Fig. 3c). Based on the American College of Medical Genetics and Genomics-Association for Molecular Pathology (ACMG-AMP) guidelines [37], we also reclassified the variant into the "Likely Pathogenic" variant. The human variant and phenotypes were reported to ClinVar (accession number: SUB8886761) and Leiden Open Variation Database [38] (LOVD; individual ID: 00326505).

\section{Discussion}

Neurodevelopmental disorders can affect the growth and development of the central nervous system, resulting in abnormal brain function that in turn may impact learning ability, self-control, emotion, memory, and motor skills [39]. Both genetic and environmental factors may take part in a substantial role in these sorts of conditions [39]. 
Neurodevelopmental disorders could have great intellectual, emotional, physical, and economic consequences for affected individuals, their families, social groups, and communities [40].

Consistently, it has been identified that the PLAA mutations are responsible for NDMSBA which is a rare neurodevelopmental disorder wherein the patients experience many neurological and non-neurological symptoms [3] (Table 1). 
Table 1

summary of clinical features

\begin{tabular}{|c|c|c|c|c|c|c|}
\hline Group & $\begin{array}{l}\text { Most } \\
\text { important } \\
\text { features }\end{array}$ & Hall et al. & Zaccai et al. & Dai et al. & $\begin{array}{l}\text { Present } \\
\text { case }\end{array}$ & Total \\
\hline \multirow{7}{*}{$\begin{array}{l}\text { Demographic } \\
\text { features }\end{array}$} & origin & Pakistani & Israeli & Chinese & Iranian & - \\
\hline & $\begin{array}{l}\text { No. affected } \\
\text { individuals }\end{array}$ & 8 & 7 & 2 & 1 & 18 \\
\hline & Male: female & $6: 2$ & $5: 2$ & $1: 1$ & $M$ & $13: 5$ \\
\hline & \multirow[t]{2}{*}{$\begin{array}{l}\text { PLAA mutant } \\
\text { genotype }\end{array}$} & \multirow[t]{2}{*}{$\begin{array}{l}\text { c.68G > T p. } \\
\text { (Gly23Val) } \\
\text { c.68dupG p. } \\
\text { (Leu24profs*55) }\end{array}$} & \multirow[t]{2}{*}{$\begin{array}{l}\text { c. } 2254 \mathrm{C}>\mathrm{T} \\
\text { p. } \\
\text { (Leu754Phe) }\end{array}$} & $\begin{array}{l}\text { c.829T > C } \\
\text { p. } \\
\text { (Cys277Arg) }\end{array}$ & \multirow[t]{2}{*}{$\begin{array}{l}\text { c. } 2264 A>G \\
\text { p. } \\
\text { (Asp755Gly) }\end{array}$} & \multirow[t]{2}{*}{-} \\
\hline & & & & $\begin{array}{l}\text { c. } 1049 \mathrm{~A}>\mathrm{T} \\
\text { p. } \\
\text { (Glu350Val) }\end{array}$ & & \\
\hline & Age of onset & $\begin{array}{l}\text { At birth (1st } \\
\text { week) }\end{array}$ & 2-4 month & 22-24 days & At birth & - \\
\hline & Age of death & 12 days -6 years & $\begin{array}{l}\text { Live at 2-34 } \\
\text { years }\end{array}$ & $\begin{array}{l}\text { Live at } 30 \\
\text { month-3 } \\
\text { years }\end{array}$ & $\begin{array}{l}\text { Alive (3 } \\
\text { years) }\end{array}$ & - \\
\hline \multirow[t]{8}{*}{$\begin{array}{l}\text { Brain } \\
\text { abnormalities }\end{array}$} & $\begin{array}{l}\text { Progressive } \\
\text { microcephaly }\end{array}$ & $8 / 8$ & $7 / 7$ & NK/YES & YES & $17 / 18(94.4 \%)$ \\
\hline & $\begin{array}{l}\text { Enlargement } \\
\text { of ventricles }\end{array}$ & $1 / 1$ & $3 / 3$ & $1 / 1$ & YES & $6 / 6(100 \%)$ \\
\hline & $\begin{array}{l}\text { White matter } \\
\text { atrophy }\end{array}$ & UK & $5 / 5$ & $1 / 1$ & YES & $6 / 6(100 \%)$ \\
\hline & $\begin{array}{l}\text { Abnormal } \\
\text { cortical } \\
\text { gyration }\end{array}$ & $6 / 6$ & $1 / 1$ & $1 / 1$ & YES & $9 / 9(100 \%)$ \\
\hline & $\begin{array}{l}\text { Thin corpus } \\
\text { callosum }\end{array}$ & $5 / 5$ & $7 / 7$ & UK & YES & $13 / 13(100 \%)$ \\
\hline & Lissencephaly & UK & UK & UK & YES & $1 / 1(100 \%)$ \\
\hline & seizure & $6 / 6$ & $3 / 7$ & $2 / 2$ & NO & $11 / 16(68.7 \%)$ \\
\hline & pachygyria & $1 / 8$ & UK & UK & YES & $2 / 8(25 \%)$ \\
\hline \multirow[t]{4}{*}{$\begin{array}{l}\text { Structural } \\
\text { abnormalities }\end{array}$} & $\begin{array}{l}\text { Craniofacial } \\
\text { deformity }\end{array}$ & $8 / 8$ & $1 / 1$ & $1 / 1$ & YES & 10/11(90\%) \\
\hline & Micrognathias & $1 / 8$ & UK & UK & YES & $2 / 9(22.2 \%)$ \\
\hline & High palate & $2 / 5$ & UK & $1 / 1$ & YES & $3 / 8(37.5 \%$ \\
\hline & Low set ears & $3 / 5$ & UK & $1 / 1$ & YES & $4 / 7(57 \%)$ \\
\hline \multirow{4}{*}{$\begin{array}{l}\text { Eye } \\
\text { abnormalities }\end{array}$} & Nystagmus & $4 / 4$ & $1 / 1$ & $1 / 1$ & YES & $7 / 7(100 \%)$ \\
\hline & strabismus & UK & UK & UK & YES & $1 / 1(100 \%)$ \\
\hline & Hypertelorism & UK & UK & UK & YES & $1 / 1(100 \%)$ \\
\hline & Optic atrophy & $4 / 5$ & $1 / 1$ & UK & YES & 6/7(85\%) \\
\hline
\end{tabular}




\begin{tabular}{|lllllll|}
\hline Group & $\begin{array}{l}\text { Most } \\
\text { important } \\
\text { features }\end{array}$ & Hall et al. & Zaccai et al. & Dai et al. & $\begin{array}{l}\text { Present } \\
\text { case }\end{array}$ & Total \\
\hline $\begin{array}{l}\text { Behavioral } \\
\text { problems }\end{array}$ & $\begin{array}{l}\text { Failure to } \\
\text { thrive }\end{array}$ & $8 / 8$ & $7 / 7$ & $2 / 2$ & YES & $18 / 18(100 \%)$ \\
\hline $\begin{array}{l}\text { Cognitive and } \\
\text { motor } \\
\text { impairment }\end{array}$ & $8 / 8$ & $7 / 7$ & $2 / 2$ & YES & $18 / 18(100 \%)$ \\
& $\begin{array}{l}\text { Poor sucking } \\
\text { and } \\
\text { swallowing }\end{array}$ & $4 / 4$ & UK & $2 / 2$ & YES & $7 / 7(100 \%)$ \\
\hline $\begin{array}{l}\text { Could not fix } \\
\text { and follow }\end{array}$ & $3 / 3$ & UK & $2 / 2$ & YES & $6 / 6(100 \%)$ \\
\hline \begin{tabular}{l} 
drooling \\
\hline
\end{tabular} & UK & UK & UK & YES & $1 / 1(100 \%)$ \\
\hline
\end{tabular}

The PLAA gene encodes PLAP protein that is highly expressed in the brain, nerve, and skeletal tissues [41]. The PLAA gene contains 14 coding exons, which yields to 2 coding transcripts that the canonical transcript translated to a 408 amino acid protein. PLAP has been shown as a contributing factor to various important biological mechanisms. This protein consists of three conserved domains including a seven-bladed WD40 beta-propeller, PLAA family ubiquitinbinding domain (PFU) in central, and a PUL domain [42]. Ubiquitin-dependent molecular chaperone p97, also known as Transitional Endoplasmic Reticulum ATPase (TER ATPase) or Valosin-Containing Protein (VCP), is an AAA ATPase that is critical for protein turnover and degradation [43]. The C-terminus of the p97 chaperone, which interacts with the PUL domain, has a crucial role in folding/unfolding substrate proteins [44]. PLAP, e.g. PLA1 and PLA2, also functions as a regulator for the activation and production of phospholipases [45]. Activated PLA2 hydrolyses membrane phospholipids into arachidonic acid that is per se used as a substrate to produce leukotrienes and prostaglandins [46].

Whole-exome Sequencing has been developed into an efficient and cost-effective tool to identify new variants and genes for rare Mendelian disorders [47]. A shred of accumulating evidence about the clinical and mutational spectrum of known and unknown diseases can be ascribed to these techniques [47]. In this study, we subjected a 2.5-year-old Iranian boy, who was referred due to neurological and developmental delay, to the paired-end WES that revealed a novel homozygous missense variant- NM_001031689.3:c.2264A > G;p.(Asp755Gly)-in the PUL domain of the PLAA gene in association with the NDMSBA disease. Using different in silico tools, we also provided evidence approving the contributory role of this variant to NDMSBA. To date, only 5 families (17 patients) and a total of 5 mutations in the PLAA gene have been reported [48-50]. Our reported variant is the 6th one that has been identified in this gene in association with NDMSBA and also the second one in the PUL domain of PLAP.

PUL domain consists of 15 tightly packed a-helices that form a structure called 'Armadillo domain' that creates a single rigid structure found in several proteins such as importin-a, $\beta$-catenins, and Hsp70 [51]. Such Armadillo repeats form banana-shaped domains that are important for the binding of other molecules [51]. The C-terminus of p97 binds to such Armadillo repeats, which is important for releasing the denatured proteins in the endoplasmic reticulum-associated protein degradation from ER and bring them to proteasome [52]. Both mutations in Hall's [50] and Dai's [48] reports were located in the ubiquitin-biding domain of PLAP that plays a crucial role in PLAA's ubiquitin signaling functions during synapse development and plasticity, known as the WD40 domain. However, Zaccai et al. showed that the mutation was located in the PUL domain [49]. In our case, the mutation is also located in such a domain that mediates binding to p97. In addition to the common phenotype of the NDMSBA condition, we observed some novel clinical features like hypertelorism, strabismus and end-gaze nystagmus in the patient; the result of the brain MRI also indicated extensive secondary deep white matter signal changes, which can be considered as the novel imaging manifestation regarding the 
severity of white matter signal changes. We believe that the intensity of clinical manifestations is due to the mutated domain-PUL domain.

Although the case reported in 2017 by Hall et al. had the most severe clinical symptoms, the MRI result did not reveal any abnormality in the brain white matter. By literature reviewing, we found that in cases affected by NDMSBA, brain white matter atrophy was prominent in the MRI, while secondary deep and subcortical white matter signal changes had not been observed in any affected individuals. Furthermore, in the last case reported by Dai et al. in 2019, one Chinese family with two affected individuals was studied [2]; using brain MRI that had been performed on the proband at the age of 23 days, no abnormality in the brain white matter was detected. Regarding our case, brain white matter signal changes were a novel finding in brain MRI findings with PLAA gene mutation, which has not been reported yet. Beyond that, strabismus, hypertelorism, were detected that had not been identified in any NDMSBA cases.

In silico analysis showed that the novel variant-p.(Asp755Gly)-may make PLAP unstable, a hypothesis which was postulated using I-Mutant $3.0 \operatorname{sever}(\Delta \Delta \mathrm{G}<0)$. The size difference between wild-type and mutant residue increase this notion that the new residue is not in the correct position to make the same hydrogen bond as the original wild-type residue did; also, Glycine is very flexible and can disturb the required rigidity of the protein at this position which may affect the function of the protein. Furthermore, conservational analysis in both nucleotide and amino acid levels, showed that the affected residue is highly conserved in primates-the animals with a higher rate of cognitive and brain function [47].

Taken together, it can be suggested that p.(Asp755Gly) may reduce the stability or proper function of the PLAP. Regarding the importance of neurodevelopmental disorders, however, more functional studies are needed to understand the exact impact of p.(Asp755Gly) on NDMSBA. We are optimistic that such studies will help us to know better brain development in the future.

\section{Conclusions}

In sum, by using paired-end WES, we reported $P L A A$ as the gene implicated in NDMSBA in a male Iranian patient. To our knowledge, this study reports the 6th variant of the PLAA gene worldwide which shows some novel clinical features such as, secondary deep white matter changes, strabismus, hypertelorism, and drooling. Before being used in genetic counseling, we strongly recommend doing functional studies by using animal models to show the molecular mechanisms underlying the pathogenesis of this novel variant. We believe that the identification of novel variants in PLAA may also yield new insights into the etiology of NDMSBA.

\section{Methods}

\section{Subjects and Ethics consideration}

We enrolled three members of a consanguineous Iranian family. The proband was a 2.5 -year-old boy suspected to NDMSBA with initial complaints of neurological and developmental delay. His parents were first-cousins who had 2 offspring one healthy and an affected sibling. The study protocol was approved by the local medical ethics committee of Tarbiat Modares University, Tehran, Iran. Written consent was achieved from all individuals present in this study and their legal representatives. They also were informed that all clinical, neuroimaging, and WES data would be used only for scientific research and not for any other purposes. All of the patient's clinical information and medical histories were collected at the Department of Medical Genetics, DeNA Laboratory, Tehran, Iran and Myelin Disorders Clinic, Pediatric Neurology Division, Tehran University of Medical Sciences, Tehran, Iran.

\section{DNA extraction and Whole-exome sequencing}

Page $8 / 16$ 
Genomic DNA was extracted from the whole blood of the proband and available family members using the standard

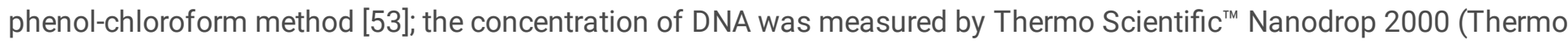
Fisher Scientific, Wilmington, DE, USA). Subsequently, around $2 \mu \mathrm{g}$ of proband's genomic DNA was subjected to pairedend WES as previously reported [47]. The DNA sample was sequenced on an Illumina HiSeq 4000 to obtain an average coverage depth of $\sim 100 \times$. Bioinformatics analyses and filtering steps were taken forward according to the previous studies $[47,54]$.

In order to validate the candidate variant and survey the segregation, Sanger sequencing in forward and reverse direction was carried out. To this end, the exon and the flanking intronic regions were amplified using PCR between the primers 5 GCCAGGCAGGACAAAAACTC-3' and 5'-TTTCTGTTTCCCCTCCCCAC-3', designed using Primer3.0 plus online tool [55]. Finally, all sequences were analyzed using CodonCode aligner software version 6.0.2 (CodonCode Corp., Centerville, MA).

\section{Prediction of single point variation on protein stability}

The non-synonymous substitutions do not always have an equal effect on protein folding and function [56]. The impact can be defined by the effects of variants on the protein folding structure and then stability [57]. In order to predict how the reported variant-p.(Asp755Gly) -affects protein stability, the I-Mutant 3.0 was utilized. This web-based tool (http://gpcr2.biocomp.unibo.it/ emidio/I-Mutant3.0) predicts the effect of a single nonsynonymous point mutation on protein stability from protein sequence by computing the $\Delta \Delta \mathrm{G}$ values of protein variant [35]. Moreover, the l-Mutant3.0 is trained to predict the thermodynamic free energy change upon single point variations in protein sequences. Furthermore, the DynaMut server [58] was used to predict the impact of the mutation on protein flexibility, conformation, and stability.

\section{Three-dimensional structure modeling}

The identified variant, p. (Asp755Gly), is located within the PUL domain of PLAP. Using ScanProsite [59], the protein families and domains were analyzed, and also sequence alignments were recruited using ClustalW (http://www.ebi.ac.uk/clustalw). The PDB structure file was built based on the Phyre2 [33] and SWISS-MODEL [34], consequently, the novel variant's possible effects on PLAP and the protein structures were depicted by PyMOL $[36,60]$.

\section{Abbreviations}

NDMSBA: Neurodevelopmental disorder with progressive microcephaly, spasticity, and brain anomalies

PLAA: Phospholipase A2-activating

PLAP: Phospholipase A-2-activating protein

WES: Whole exome sequencing

HC: head circumference

DDST: Denver Developmental Screening Test

MRI: magnetic resonance imaging

gnomAD: genome aggregation database

SIFT: Sorting Intolerant from Tolerant

ACMG-AMP: American College of Medical Genetics and Genomics-Association for Molecular Pathology

PFU: PLAA family ubiquitin-binding domain 
TER ATPase: Transitional Endoplasmic Reticulum ATPase

\section{Declarations}

\section{Ethics approval and consent to participate}

The study protocol was approved by the local medical ethics committee of Tarbiat Modares University, Tehran, Iran. The written informed consent was received from each patient and guardian and they also provided a signed written consent form to publish all personal and medical details. All methods were performed in accordance with the relevant guidelines and regulations of the Declaration of Helsinki.

\section{Consent for publication}

The informed consent to publish was obtained from each patient and guardian.

\section{Availability of data and materials}

The datasets used and/or analyzed during the current study are available from the corresponding author on reasonable request. The variant and pertinent phenotypes caused by a mutation in RAB3GAP1 are accessible at ClinVar (SUB8886761) and Leiden Open Variation Database (LOVD; individual number: 00326505).

\section{Competing interests}

The authors declare that they have no competing interests.

\section{Funding}

Not applicable.

\section{Authors' Contributions}

ART and MG conceived and planned the experiments. AD carried out the experiments. $M G, A D$, and ER planned and carried out the data analyses. FB contributed to sample preparation. $A D, E R$, and MG contributed to the interpretation of the results. AD took the lead in writing the manuscript. ART and MR carried out clinical analyses. All authors provided critical feedback and helped shape the research, analysis, and manuscript.

\section{Acknowledgments}

We thank the family members for their willingness and contribution.

\section{References}

1. Falik Zaccai TC, Savitzki D, Zivony-Elboum Y, Vilboux T, Fitts EC, Shoval Y, et al. Phospholipase A2-activating protein is associated with a novel form of leukoencephalopathy. Brain. 2017;140(2):370-86. 
2. Dai C, Zeng S, Tan Z, Yang X, Du J, Lu G, et al. Neurodevelopmental disorder with progressive microcephaly, spasticity, and brain anomalies in China caused by novel mutations of PLAA. Clin Genet. 2019;96(4):380-1.

3. Hall EA, Nahorski MS, Murray LM, Shaheen R, Perkins E, Dissanayake KN, et al. PLAA mutations cause a lethal infantile epileptic encephalopathy by disrupting ubiquitin-mediated endolysosomal degradation of synaptic proteins. The American Journal of Human Genetics. 2017;100(5):706-24.

4. Pontén F, Jirström K, Uhlen M. The Human Protein Atlas-a tool for pathology. The Journal of Pathology: A Journal of the Pathological Society of Great Britain and Ireland. 2008;216(4):387-93.

5. Kudo I, Murakami M. Phospholipase A2 enzymes. Prostaglandins Other Lipid Mediat. 2002;68:3-58.

6. Zhang F, Sha J, Wood TG, Galindo CL, Garner HR, Burkart MF, et al. Alteration in the activation state of new inflammation-associated targets by phospholipase A2-activating protein (PLAA). Cell Signal. 2008;20(5):844-61.

7. Shi W, Ding R, Zhou PP, Fang Y, Wan R, Chen Y, et al. Coordinated actions between p97 and Cullin-RING ubiquitin ligases for protein degradation. Cullin-RING Ligases and Protein Neddylation: Springer; 2020. p. 61-78.

8. Hänzelmann P, Schindelin H. The interplay of cofactor interactions and post-translational modifications in the regulation of the AAA+ ATPase p97. Frontiers in molecular biosciences. 2017;4:21.

9. Jain BP. Genome Wide Analysis of WD40 Proteins in Saccharomyces cerevisiae and Their Orthologs in Candida albicans. The Protein Journal. 2019;38(1):58-75.

10. Park S-R, Hwang J, Kim M. The Arabidopsis WDR55 is positively involved in ABA-mediated drought tolerance response. Plant Biotechnology Reports. 2020:1-12.

11. Valnegri P, Huang J, Yamada T, Yang Y, Mejia LA, Cho HY, et al. RNF8/UBC13 ubiquitin signaling suppresses synapse formation in the mammalian brain. Nature communications. 2017;8(1):1-15.

12. Hyer ML, Milhollen MA, Ciavarri J, Fleming P, Traore T, Sappal D, et al. A small-molecule inhibitor of the ubiquitin activating enzyme for cancer treatment. Nat Med. 2018;24(2):186.

13. Wu J, Xia L, Yao X, Yu X, Tumas KC, Sun W, et al. The E3 ubiquitin ligase MARCH1 regulates antimalaria immunity through interferon signaling and T cell activation. Proceedings of the National Academy of Sciences. 2020;117(28):16567-78.

14. Lei X, Zhang L, Li Z, Ren J. Astragaloside IV/IncRNA-TUG1/TRAF5 signaling pathway participates in podocyte apoptosis of diabetic nephropathy rats. Drug Des Devel Ther. 2018;12:2785.

15. Le Guerroué F, Youle RJ. Ubiquitin signaling in neurodegenerative diseases: an autophagy and proteasome perspective. Cell Death Differ. 2020:1-16.

16. Upadhyay A, Amanullah A, Mishra R, Kumar A, Mishra A. Lanosterol suppresses the aggregation and cytotoxicity of misfolded proteins linked with neurodegenerative diseases. Mol Neurobiol. 2018;55(2):1169-82.

17. HEYDARI ST, EMAM GF, Amini M. INFANTS'GROWTH CHARTS IN JAHROM, IRAN. 2009.

18. Fallah R, Ehsani-Khanghah Y, Motamed N. Evaluation of Head circumference index in children under 18 months and its associated factors in Zanjan city: A Retrospective Cohort. Int J Pediatr. 2020:12805-13.

19. Sherry ST, Ward M-H, Kholodov M, Baker J, Phan L, Smigielski EM, et al. dbSNP: the NCBI database of genetic variation. Nucleic Acids Res. 2001;29(1):308-11.

20. Smigielski EM, Sirotkin K, Ward M, Sherry ST. dbSNP: a database of single nucleotide polymorphisms. Nucleic Acids Res. 2000;28(1):352-5.

21. Li H, Handsaker B, Wysoker A, Fennell T, Ruan J, Homer N, et al. 1000 Genome Project Data Processing Subgroup. 2009. The sequence alignment/map format and samtools. Bioinformatics. 2009;25(16):2078-9.

22. Server EV. NHLBI GO exome sequencing project (ESP). Exome Variant Server Seattle, WA; 2011.

23. Karczewski K, Francioli L. The genome aggregation database (gnomAD). MacArthur Lab. 2017.

Page $11 / 16$ 
24. Vaser R, Adusumalli S, Leng SN, Sikic M, Ng PC. SIFT missense predictions for genomes. Nat Protoc. 2016;11(1):1.

25. Adzhubei I, Jordan DM, Sunyaev SR. Predicting functional effect of human missense mutations using PolyPhen-2. Current protocols in human genetics. 2013;76(1):7.20. 1-7.. 41.

26. Schwarz JM, Rödelsperger C, Schuelke M, Seelow D. MutationTaster evaluates disease-causing potential of sequence alterations. Nature methods. 2010;7(8):575-6.

27. Landrum MJ, Lee JM, Benson M, Brown G, Chao C, Chitipiralla S, et al. ClinVar: public archive of interpretations of clinically relevant variants. Nucleic Acids Res. 2016;44(D1):D862-D8.

28. Yang H, Robinson PN, Wang K. Phenolyzer: phenotype-based prioritization of candidate genes for human diseases. Nature methods. 2015;12(9):841-3.

29. Mishima H, Suzuki H, Doi M, Miyazaki M, Watanabe S, Matsumoto T, et al. Evaluation of Face2Gene using facial images of patients with congenital dysmorphic syndromes recruited in Japan. J Hum Genet. 2019;64(8):789-94.

30. Fattahi Z, Beheshtian M, Mohseni M, Poustchi H, Sellars E, Nezhadi SH, et al. Iranome: A catalog of genomic variations in the Iranian population. Hum Mutat. 2019;40(11):1968-84.

31. Choi Y, Chan AP. PROVEAN web server: a tool to predict the functional effect of amino acid substitutions and indels. Bioinformatics. 2015;31(16):2745-7.

32. Frankish A, Harrow J. GENCODE pseudogenes. Pseudogenes: Springer; 2014. p. 129-55.

33. Kelley LA, Mezulis S, Yates CM, Wass MN, Sternberg MJ. The Phyre2 web portal for protein modeling, prediction and analysis. Nat Protoc. 2015;10(6):845.

34. Bienert S, Waterhouse A, de Beer TA, Tauriello G, Studer G, Bordoli L, et al. The SWISS-MODEL Repository-new features and functionality. Nucleic Acids Res. 2017;45(D1):D313-D9.

35. Capriotti E, Fariselli P, Casadio R. I-Mutant2. 0: predicting stability changes upon mutation from the protein sequence or structure. Nucleic Acids Res. 2005;33(suppl_2):W306-W10.

36. DeLano WL. Pymol: An open-source molecular graphics tool. CCP4 Newsletter on protein crystallography. 2002;40(1):82-92.

37. Biesecker LG, Harrison SM. The ACMG/AMP reputable source criteria for the interpretation of sequence variants. Genet Med. 2018;20(12):1687.

38. Fokkema IF, Taschner PE, Schaafsma GC, Celli J, Laros JF, den Dunnen JT. LOVD v. 2.0: the next generation in gene variant databases. Hum Mutat. 2011;32(5):557-63.

39. Ullman MT, Pullman MY. A compensatory role for declarative memory in neurodevelopmental disorders. Neurosci Biobehav Rev. 2015;51:205-22.

40. Miller A, Shen J, Mâsse LC. Child functional characteristics explain child and family outcomes better than diagnosis: population-based study of children with autism or other neurodevelopmental disorders/disabilities. Health Rep. 2016;27(6):9.

41. Yagami T, Yamamoto $Y$, Koma $H$. The role of secretory phospholipase $A 2$ in the central nervous system and neurological diseases. Mol Neurobiol. 2014;49(2):863-76.

42. Pashkova N, Gakhar L, Winistorfer SC, Yu L, Ramaswamy S, Piper RC. WD40 repeat propellers define a ubiquitinbinding domain that regulates turnover of $F$ box proteins. Mol Cell. 2010;40(3):433-43.

43. Zhong X, Shen Y, Ballar P, Apostolou A, Agami R, Fang S. AAA ATPase p97/valosin-containing protein interacts with gp78, a ubiquitin ligase for endoplasmic reticulum-associated degradation. J Biol Chem. 2004;279(44):45676-84.

44. Yeung HO, Kloppsteck P, Niwa H, Isaacson RL, Matthews S, Zhang X, et al. Insights into adaptor binding to the AAA protein p97. Biochem Soc Trans. 2008;36(1):62-7.

45. Aoki J, editor Mechanisms of lysophosphatidic acid production. Semin Cell Dev Biol; 2004: Elsevier. 
46. Esmaeilzadeh-Gharehdaghi E, Razmara E, Bitaraf A, Jamshidi A, Mahmoudi M, Garshasbi M. Functional Analysis of RELN S2486G Mutation and its Contribution to Pathogenesis of Ankylosing Spondylitis. Archives of Iranian Medicine (AIM). 2020;23(10).

47. Razmara E, Azimi H, Tavasoli AR, Fallahi E, Sheida SV, Eidi M, et al. Novel neuroclinical findings of autosomal recessive primary microcephaly 15 in a consanguineous Iranian family. Eur J Med Genet. 2020;63(12):104096.

48. Dai C, Zeng S, Tan Z, Yang X, Du J, Lu G, et al. Neurodevelopmental disorder with progressive microcephaly, spasticity, and brain anomalies in China caused by novel mutations of PLAA. Clin Genet. 2019;96(4):380-1.

49. Falik Zaccai TC, Savitzki D, Zivony-Elboum Y, Vilboux T, Fitts EC, Shoval Y, et al. Phospholipase A2-activating protein is associated with a novel form of leukoencephalopathy. Brain. 2017;140(2):370-86.

50. Hall EA, Nahorski MS, Murray LM, Shaheen R, Perkins E, Dissanayake KN, et al. PLAA Mutations Cause a Lethal Infantile Epileptic Encephalopathy by Disrupting Ubiquitin-Mediated Endolysosomal Degradation of Synaptic Proteins. Am J Hum Genet. 2017;100(5):706-24.

51. Hatzfeld M. The armadillo family of structural proteins. International review of cytology. 1999;186:179-224.

52. Yeung HO, Kloppsteck P, Niwa H, Isaacson RL, Matthews S, Zhang X, et al. Insights into adaptor binding to the AAA protein p97. 2008;36(1):62-7.

53. Köchl S, Niederstätter H, Parson W. DNA extraction and quantitation of forensic samples using the phenolchloroform method and real-time PCR. Forensic DNA typing protocols: Springer; 2005. p. 13-29.

54. Aryan H, Razmara E, Farhud D, Zarif-Yeganeh M, Zokaei S, Hassani SA, et al. Novel imaging and clinical phenotypes of CONDSIAS disorder caused by a homozygous frameshift variant of ADPRHL2: a case report. BMC Neurol. 2020;20(1):1-11.

55. Untergasser A, Nijveen H, Rao X, Bisseling T, Geurts R, Leunissen JA. Primer3Plus, an enhanced web interface to Primer3. Nucleic Acids Res. 2007;35(suppl_2):W71-W4.

56. Hassan MS, Shaalan A, Dessouky M, Abdelnaiem AE, ElHefnawi M. Evaluation of computational techniques for predicting non-synonymous single nucleotide variants pathogenicity. Genomics. 2019;111(4):869-82.

57. Ancien F, Pucci F, Godfroid M, Rooman M. Prediction and interpretation of deleterious coding variants in terms of protein structural stability. Sci Rep. 2018;8(1):1-11.

58. Rodrigues $\mathrm{CH}$, Pires DE, Ascher DB. DynaMut: predicting the impact of mutations on protein conformation, flexibility and stability. Nucleic Acids Res. 2018;46(W1):W350-W5.

59. Gattiker A, Gasteiger E, Bairoch AM. ScanProsite: a reference implementation of a PROSITE scanning tool. Applied bioinformatics. 2002;1(2):107-8.

60. DeLano WL. PyMOL. 2002.

\section{Figures}




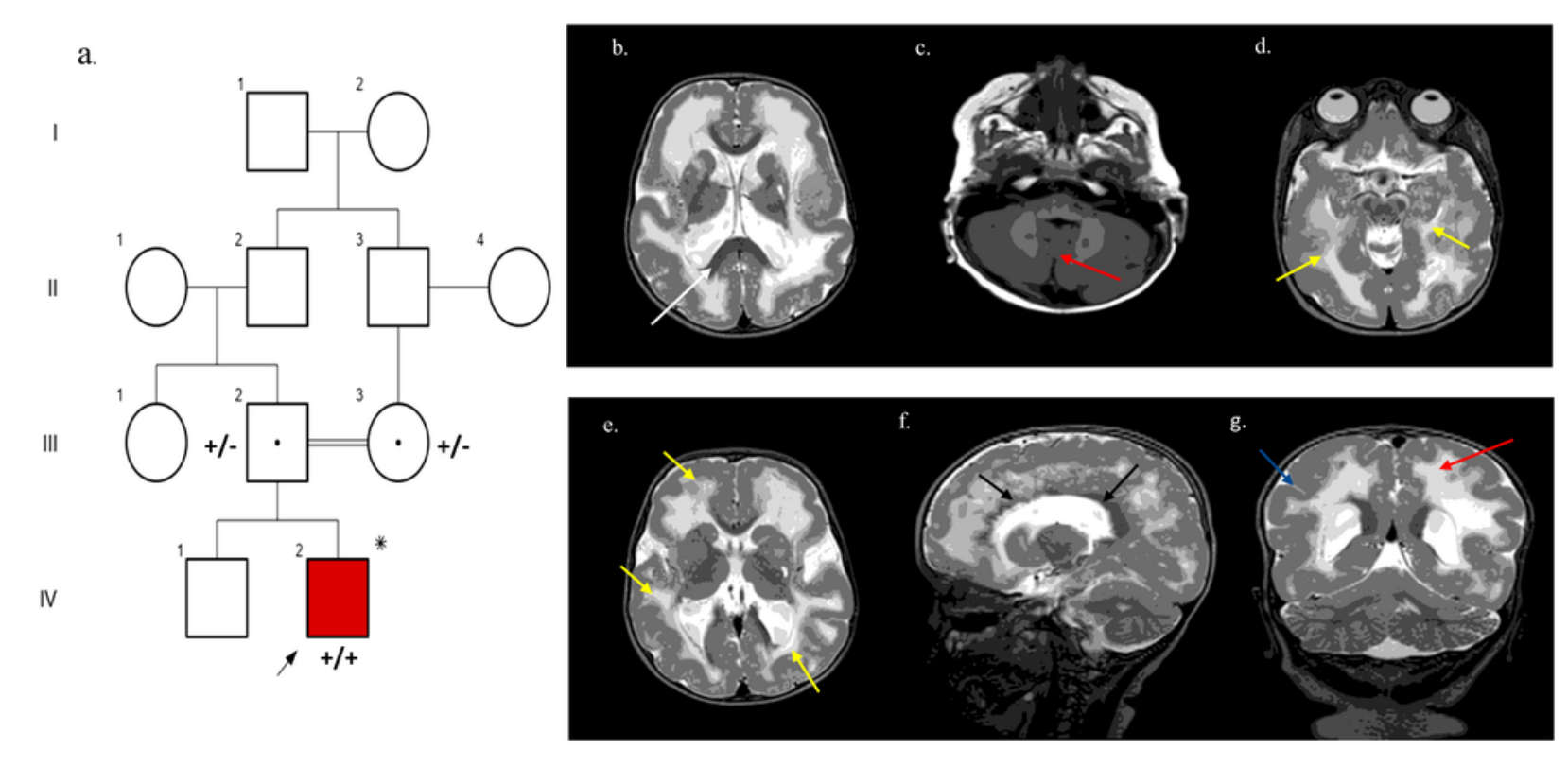

\section{Figure 1}

Pedigree and MRI findings in the family. a) Pedigree of the consanguineous Iranian family consists 4 generations. In this figure, the squares and circles indicate males and females, respectively; the black arrow shows the proband. The heterozygosity for the variant is shown by +/-, while homozygosity for the novel variant is shown by $+/+$. An asterisk (*) indicates the sample which was selected for applying whole-exome sequencing. b) Axial T2-Weighted image of brain MRI at the age of 18 months, shows extensive deep, periventricular and subcortical white matter signal changes, Lissencephay and abnormal disorganized gyral pattern and colpocephaly (white arrow). c) Axial T1-Weighted image shows mild vermis atrophy (red arrow). $d$ and e) Axial T2-Weighted images represent anomalies in myelination and deep/subcortical white matter signal changes (yellow arrows). f) Sagittal T2-Weighted image shows thin corpus callosum (black arrows). g) Coronal T2-Weighted image of brain MRI shows pachygyria/polymicrogyria (blue arrow-right side) and confirms peri-dentate white matter signal changes (red arrow). 


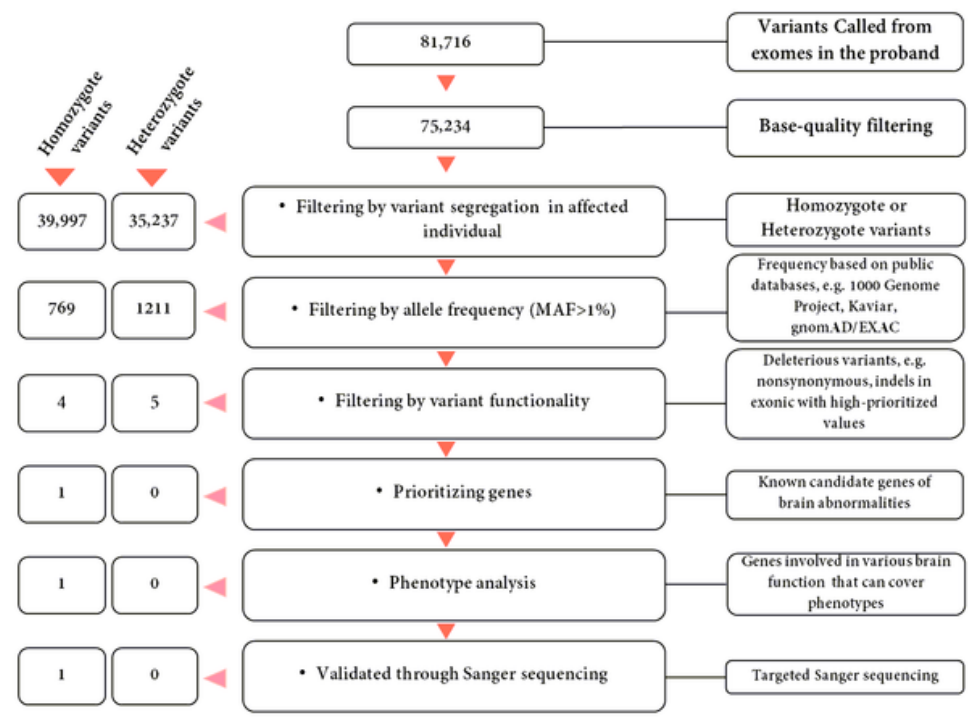

b.

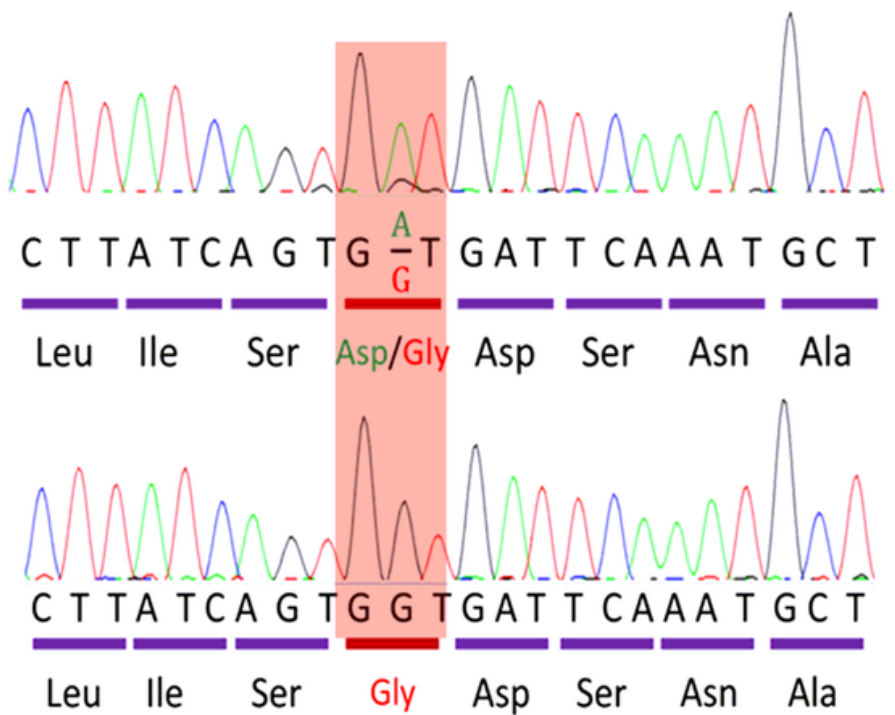

Figure 2

whole-exome sequencing data analyses and also the chromatograms. a) filtering steps that had been taken forward to analyze/filter the derived data. the data were reanalyzed by assuming the autosomal dominant inheritance, but no convincing finding was resulted in. b) chromatograms confirmed the homozygosity and heterozygosity for the novel variant in the family.

a.

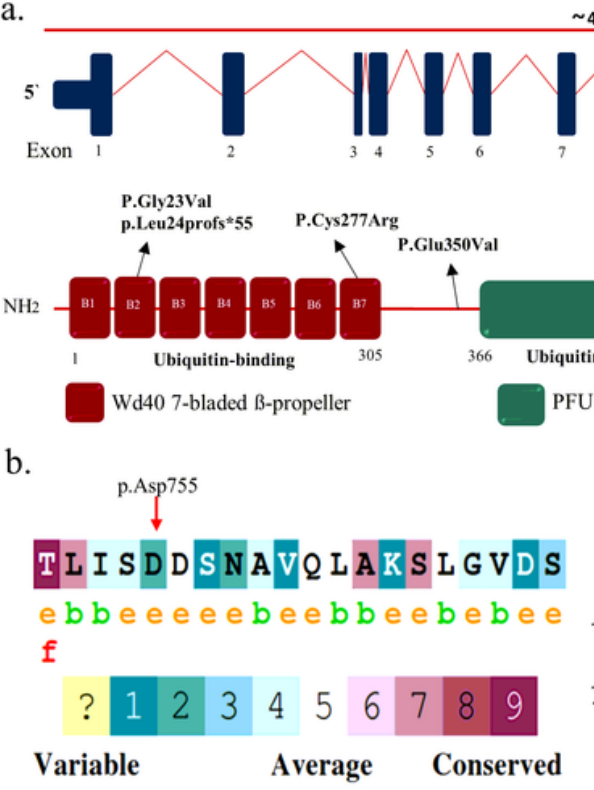

b.

- An exposed residue according to the neural-network algorithn.

$b$ - A buried residue according to the neural-network algorithm.

f - A predicted functional residue (highly conserved and exposed).

$s$ - A predicted structural residue (highly conserved and buried). $\sim 44 \mathrm{kbp}$
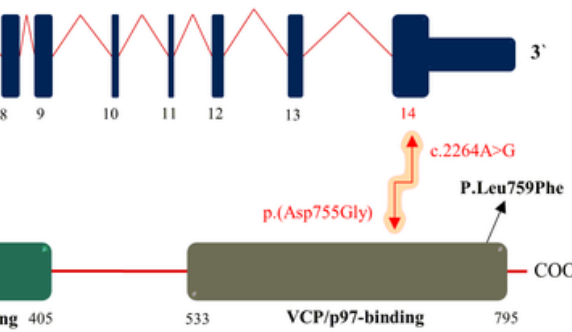

PUL domain

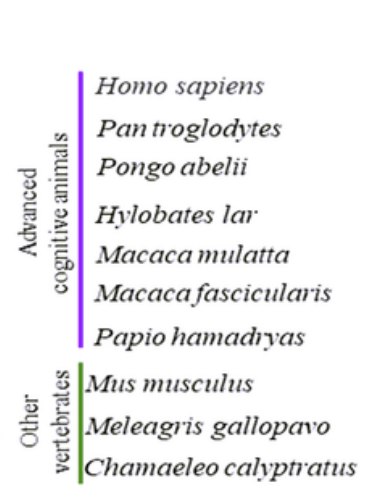

c.

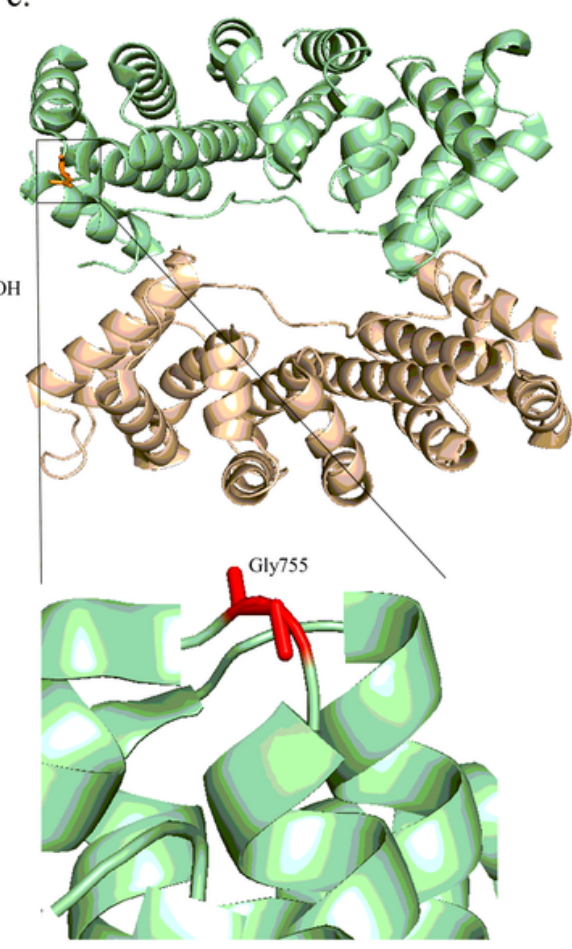

Figure 3 
a) schematic structure of the PLAA gene and the encoded protein. This gene spans $\sim 44 \mathrm{kbp}$ and the encoded protein is comprised of 795 amino acids and three different domains (are shown by different colors). The red arrow indicates the position of the variant detected in this study; the variant is located in exon 14 that per se encodes some parts of the PUL domain region of PLAP. PLAP involves three different parts: WD40 domain contains in seven-bladed beta-propeller WD40 repeats in N-terminus, the PFU domain which is in the central part of PLAP and appears to be unique to the PLAA family of proteins, and C-terminus part of the protein which directly interacts with p97, an enzyme from the AAA-ATPase family of molecular chaperones. b) The amino acid sequence of PLAP is colored based on conservation scores by the ConSurf database. ConSurf is a repository for evolutionary conservation analysis of the known/unknown protein structure according to the phylogenetic relations between homologous sequences as well as amino acid's structural and functional importance. The results show that Gly755 is located in a variable exposed region based on the comparison performed among the candidate vertebrae and invertebrates. In the nucleotide level, the '2-Way Pseudogene Annotation Set' from the UCSC genome browser indicates the high conservation of the region embarrassing the variant site in advanced cognitive animals, i.e. primates. c) The three-dimensional structure of the PLAP is shown. The structure was made using PyMOL. The mutant residue is shown by red. 